\title{
Ti:sapphire Laser Ablation of Silicon in Different Ambients
}

\author{
Miklós FÜLE ${ }^{* 1,2}$, Anett GÁRDIÁN ${ }^{* 3}$, János CSONTOS $^{* 3}$, Judit BUDAI ${ }^{* 3}$ and Zsolt TÓTH ${ }^{* 3}$ \\ ${ }^{{ }^{*}}$ Department of General and Environmental Physics, University of Szeged,H-6725 Szeged \\ Boldogasszony sgt. 6., Hungary \\ E-mail: miklos.fule@gmail.com \\ ${ }^{* 2}$ High Intensity Laser Laboratory, Department of Experimental Physics, University of Szeged, \\ H-6725 Szeged, Dóm tér 9., Hungary \\ ${ }^{* 3}$ Department of Optics and Quantum Electronics, University of Szeged, H-6725 Szeged, Dóm tér 9. \\ Hungary
}

\begin{abstract}
Titanium-sapphire laser ablation of silicon was investigated in atmospheric air, water and $20 \%$ $\mathrm{KOH}$ solution. The number of overlapping pulses was tuned between 3 and 1000 by changing the processing time. The ablated surfaces were investigated using scanning electron microscopy, X-ray microanalysis and stylus profilometry. In each ambient, plasma formation was observed during ablation. X-ray microanalysis indicated that the process starts with surface oxidation in case of air and water ambient. With increasing pulse number the surface was roughened, resulting in larger structures. Crater formation was observed in air and in water, with higher etching rate in the latter case. The depth profile of the ablation holes and grooves is rather different for this two ambient. Narrow conical grooves were observed in case of ablation in air, while flat bottom grooves were produced in water. Laser processing in $\mathrm{KOH}$ solution did not result in etching, only the native oxide layer was removed. Etching occurred in these areas, if the samples were left in $\mathrm{KOH}$ solution for a longer time. Based on these observations the material removal by fs laser pulses is attributed to evaporation and reactions of Si with radicals in the laser induced plasma, such as atomic or ionic oxygen. The application of $\mathrm{KOH}$ introduces $\mathrm{K}$ ions or atoms into the laser induced plasma, which may react faster with oxygen than with $\mathrm{Si}$, thus resulting in decrease in etching rate during laser processing.
\end{abstract}

DOI: $10.2961 / \mathrm{jlmn} .2014 .02 .0008$

Keywords: Ti:sapphire laser, ablation, silicon, plasma formation, laser oxidation, nanostructures

\section{Introduction}

Femtosecond laser ablation has received a significant attention in the last decade due to its advantages over nanosecond ablation, as e.g. reduced heat affected zone and burr-free edges. Therefore fs high power laser pulses have become widely applied in material processing, in several scientific fields and industrial applications [1]. Absorption of a fs laser pulse by a semiconductor surface take place via several steps, which have different timescales. First the electrons of the conduction band are totally freed from the lattice within 10 to $100 \mathrm{fs}$. As a next step the excited electrons and holes are redistributed via carrier - carrier and carrier - phonon scattering with a timescale of several tens of femtosecond to several picoseconds. After the lattice and the electron subsystem come to an equilibrium temperature the excess carrier removal and thermal/structural effects take place parallel. Ablation and evaporation occurs after tens of picoseconds, the plume expands from the heated surface into the ambient. Velocities up to $10^{5} \mathrm{~m} / \mathrm{s}$ were measured for silicon in air atmosphere using $10^{13} \mathrm{~W} / \mathrm{cm}^{2}$ laser intensity [2]. Cooling and resolidification of the surface appears on the ns to $\mu$ s range [3]. Ablation process is strongly influenced by the surrounding ambient and its chemical nature [4].

Ablation of silicon in vacuum [5-8], gases [7-14] and liquids [8, 12-21] has been investigated extensively. In this work the modification of surface-morphology of bulk silicon target, exposed to fs laser irradiation in air, water and
$\mathrm{KOH}$ solution ambient was studied. Our aim was to examine the evolution of the surface morphology in case of multipulse irradiation and to investigate the effect of a chemically active ambient.

\section{Experimental}

In our experiments a Ti:sapphire laser (central wavelength: $800 \mathrm{~nm}$ and pulse length: $22 \mathrm{fs}$ ) was used. The energy at the sample surface and above the liquid was kept in the experiments performed in air and water ambient at 260 $\mu \mathrm{J}$, while in case of $\mathrm{KOH}$ experiments at $330 \mu \mathrm{J}$. The repetition rate of the laser pulses were $200 \mathrm{~Hz}$. The laser beam was linearly polarized. The $\mathrm{x}$ axis of the presented electron microscope images is set parallel with the polarization direction. Before irradiation the (100) silicon wafer pieces were cleaned in ultrasonic bath under acetone and ethanol separately. The Si pieces were placed into a PTFE pot mounted on a three-axis translation stage. The beam of Ti:sapphire laser directed by a mirror onto the sample perpendicularly and it was focused by a $100 \mathrm{~mm}$ focal length lens. The surface treatment was investigated in air, under water, and $\mathrm{KOH}(20 \mathrm{wt} \%)$ solution at room temperature. The volume of the liquids was $11545 \mu$ l. When the ablation of Si was examined in liquid media, the thickness of the liquid layer above the sample surface was $6 \mathrm{~mm} \pm 5 \mu \mathrm{m}$.

To modify the energy density at the surface, the sample was moved vertically ( $\mathrm{z}$ axis), so the diameter and the irradiated area was changed. To examine the pulse number 
dependence a shutter was used to control the pulse number between 3 and 1000. The spot size and thus the fluence of the certain sample position were estimated by determining the area of irradiated spots from secondary electron scanning electron microscope images and the focusing geometry. Since the area of the modified surface is strongly influenced by non-linear absorption, plasma and liquid etching rates, therefore they can not be directly conducted to any parameter of the laser. Therefore average fluence values can be rather erroneous [22], they are used for comparison purposes only.

For the investigation of the modified surfaces we used scanning electron microscopy (SEM) and X-ray microanalysis (EDX) using a Hitachi S-4700 microscope. The depth of the ablated holes was determined by stylus profilometry (DEKTAK 8).

\section{Results}

During the ablation process an intense plasma point was observed in all ambient. The color and visibility of the plasma was strongly influenced by the chemical composition. In air from purple to yellow, in water and $\mathrm{KOH}$ solution bright yellow or white spot formed, above the treated area. The brightest plumes were produced in case of $\mathrm{KOH}$ solution. When the focal plane was above the $\mathrm{Si}$ sample despite of the formation of a plasma plume the Si surface was not affected by the pulse since the energy was mostly converted into the ambient, heating it up. Therefore in the followings the presented results belong to cases when the focal plane was inside the sample or on the surface.

\subsection{Air and water ambient}

\subsubsection{Energy density dependence}

Firstly the effect of energy density on surface morphology was examined. The variation of the sample position along the $\mathrm{z}$ axis changes the spot size and therefore the energy density, resulting in different ablated surfaces as it can be clearly seen in Fig. 1, from the corresponding profilometric data of the treated areas.

In case of air ambient (Fig. $1 \mathrm{~A}$ ) the lowest fluence values result in partial ablation of the laser treated spot. As shown by SEM images, when energy density increases the ablated surface covers the whole area of the laser spot and rougher structures are left. Finally the laser pulses of the highest energy density etch grooves on the surface, increasing its roughness. This morphological change can be followed in the profilometry results, too. In case of low energy density the surface height increases up to nearly $150 \mathrm{~nm}$. At higher laser energy densities hump-like structures are formed at the edges and groove-like structures are produced in the middle of the processed area. The appearance of humps is attributed to the deposition of the ablated material, which is direct consequence of the high pressure ambient [23].

In case of water ambient the SEM image indicates similar situation at the lowest energy density case (Fig. 1 B). At these fluence values small protrusion-like structures appear also on the surfaces. However as the energy density increases the surface morphology strongly differs from the air ambient case. The roughness also rose but the hump formation at the edges is not significant; just a little shoulder can be observed around the etched region. The absence of hump-like structures indicates that the convection of liquid ambient washes away the ablated material.
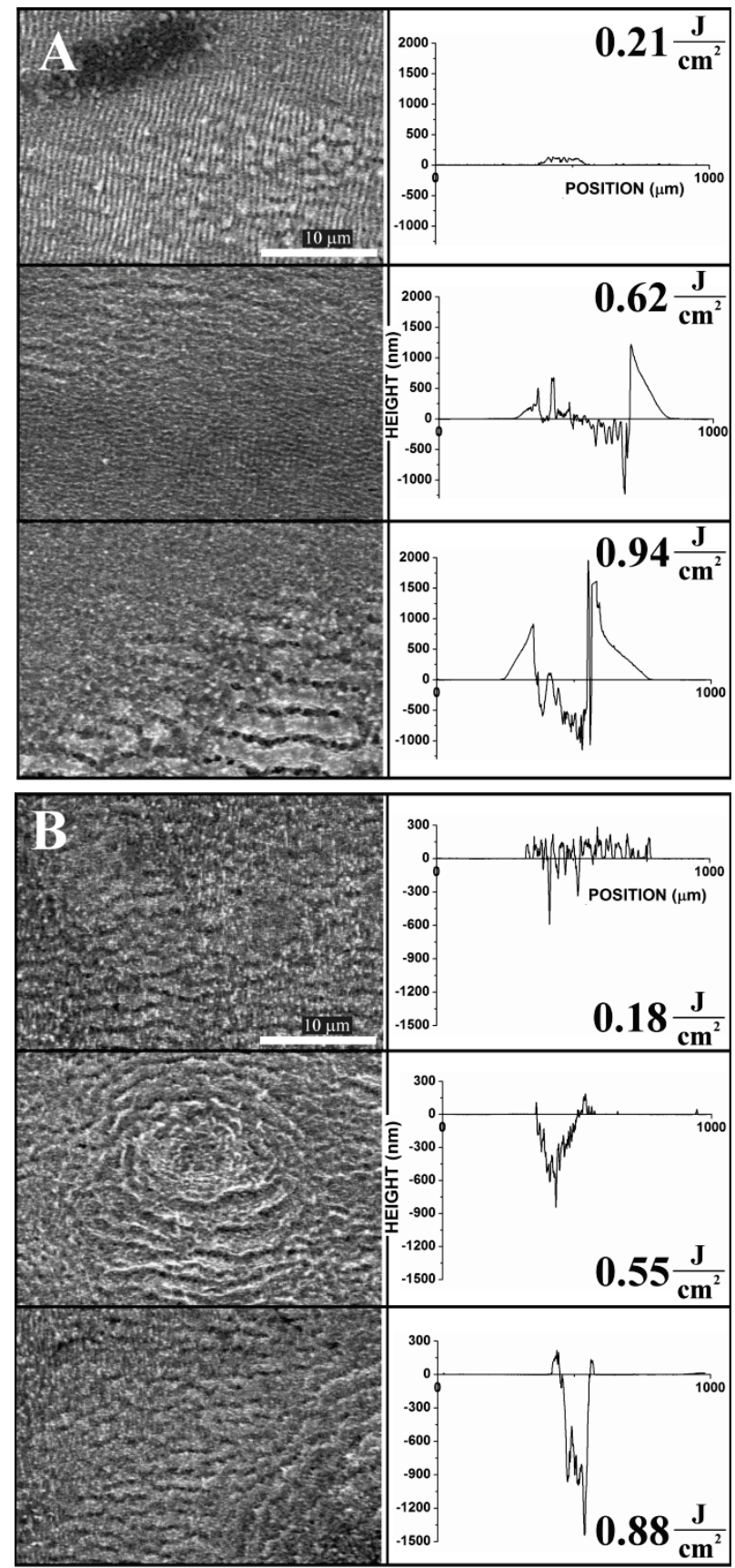

Fig. 1 SEM images of 100 pulse treated areas (left column), and corresponding profiles (right column) in A) air, and $\mathrm{B}$ ) water. The corresponding fluence values are indicated in the sub-figures.

Fig. 2 gives a summary of the profilometry measurements: the depths of the ablated holes are plotted as a function of the applied average laser fluence. Efficient material removal occurs near to $1 \mathrm{~J} / \mathrm{cm}^{2}$. However, at higher fluences (in focus position) and when the focal point is situated in the surrounding medium, removal stops and even increase in height was observed. 


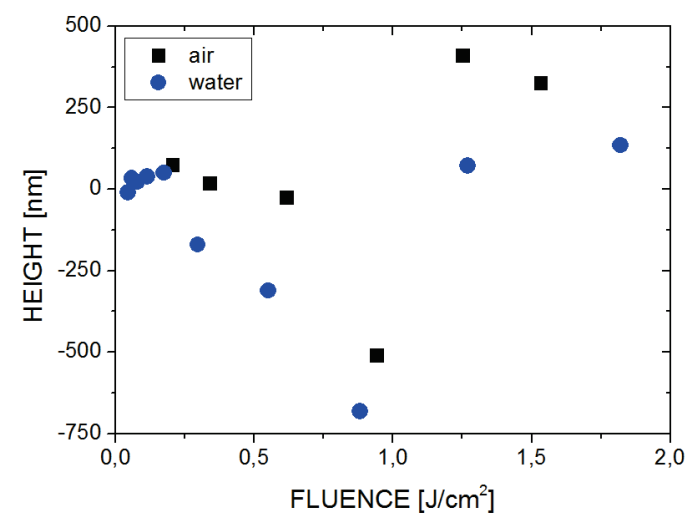

Fig. 2 Depths of ablated holes $(\mathrm{N}=100)$ as a function of fluence, determined from profilometry measurements in case of air (squares) and water (circles) ambient.

\subsubsection{Pulse number dependence}

Our following experiments targeted the examination of the pulse number dependence of the formed structures. For this examination the energy densities were 0.55 and 0.62 $\mathrm{J} / \mathrm{cm}^{2}$ for water and air, respectively. Our results concerning this examination are summarized in Fig. 3.

The left column consists of SEM images while the right column contains the corresponding profilometry results. In the SEM images the scale bar indicates $10 \mu \mathrm{m}$. The pulse number was 3,100 and 1000 from top to bottom.

Fig. 3 A shows our results concerning air ambient. It is clearly visible on the top SEM image that the surface modification has started after three laser pulses and stained surfaces are formed. With increasing pulse number, stripe-like structures have formed from the stains on the surface. These structures already have depth and in this case processing with higher pulse number starts to form a shoulder also around the treated area. The inhomogeneity of the depth of stripes is also obvious from the profilometry results. This inhomogeneity became more intense with growing pulse number. The bottom SEM image shows not just that the orientation of stripe structure rotate into parallel direction with laser polarization but large-scale structures also appear as it is clear from profilometric data also.

The situation is more interesting in case of water ambient. Applying three pulse shots on the surface under the water ambient provide also a structure without observable depth. In this case ring-like surface structures with different diameter appear in the SEM image. With increasing number of laser pulses the evolution of these structures can be observed: the structure became more complex and rough. A star-like form is added to the ring shape of the structure at the highest pulse numbers. According to the profilometry data a greater depth can also be observed here.
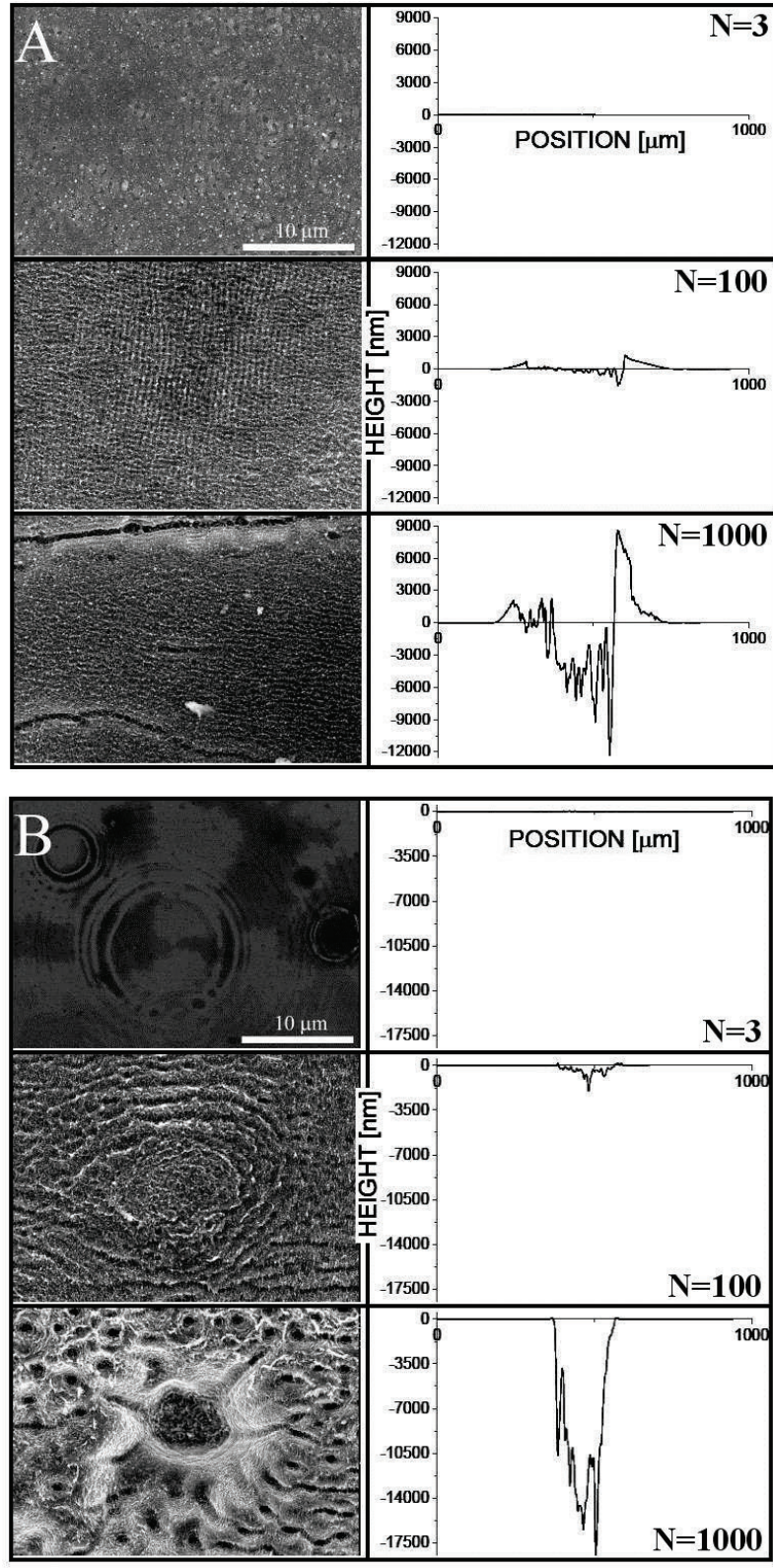

Fig. 3 SEM images (left column), and corresponding profiles (right column) taken from areas, processed with different $(3,100$ and 1000) number of pulses. The samples were prepared with $0.62 \mathrm{~J} / \mathrm{cm}^{2}$ in air (A) and $0.55 \mathrm{~J} / \mathrm{cm}^{2}$ fluence in water (B).

In Fig. 4 the average depth of the ablated holes are plotted as a function of the number of ablating pulses. The deepening of the holes follows a linear dependence for the two ambient materials. The deepest holes were etched in water. Nearly half depth of them was produced in air. 


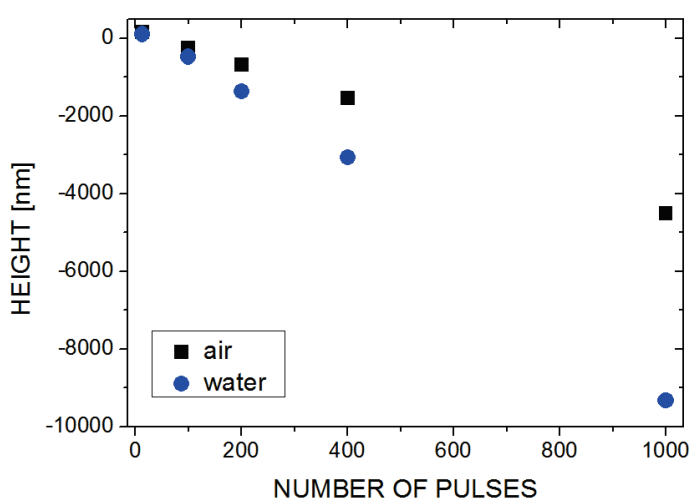

Fig. 4 Depths of ablated holes as a function of pulse number, determined from profilometry measurements in case of air (squares) and water (circles) ambient, at 0.62 and 0.55 $\mathrm{J} / \mathrm{cm}^{2}$ laser fluence, respectively.

For deeper understanding of the evolution of the surface forms EDX spectroscopy has been performed in the middle of the ablated patterns. Beside the strong Si peak, the characteristic peak of oxygen could be identified for all samples.

The X-ray excitation depth may be much larger than the surface oxide layer thickness. In order to characterize the oxygen content, the ratio of the height of the peaks of $\mathrm{O}$ and $\mathrm{Si}$ was calculated. The oxygen to silicon peak ratio as the function of number of laser pulses is presented in Fig. 5. The highest ratio belongs to the samples prepared in air ambient. In this case the oxygen to silicon ratio continuously rises with increasing number of laser pulses. Somewhat lower amount was observable in the case of laser irradiation in water. It is worth to note, that already a significant increase of $\mathrm{O}$ content was observed after some shots.

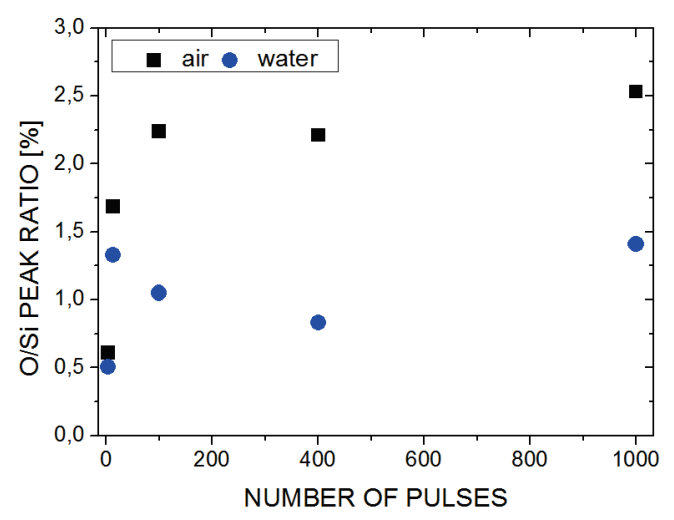

Fig. $5 \mathrm{O} / \mathrm{Si}$ ratio in EDX spectra in central areas, processed with different pulse numbers in case of air (squares) and water (circles), at 0.62 and $0.55 \mathrm{~J} / \mathrm{cm}^{2}$ laser fluence, respectively.

\subsection{KOH ambient}

Si treatment by ultrafast Ti-sapphire laser pulses in $\mathrm{KOH}$ solution is turned out more complex than treatments in air or water. Figure 6 A) and B) shows Si surfaces treated by 100 laser pulses and the corresponding depth profiles. In both cases the energy density was $0,59 \mathrm{~J} / \mathrm{cm}^{2}$. The only difference between these cases was that in the case A) the sample was removed from $\mathrm{KOH}$ shortly (3 minutes) after processing, and rinsed in distilled water, while in the case B) the sample stayed in the solution for $\sim 20$ minutes.

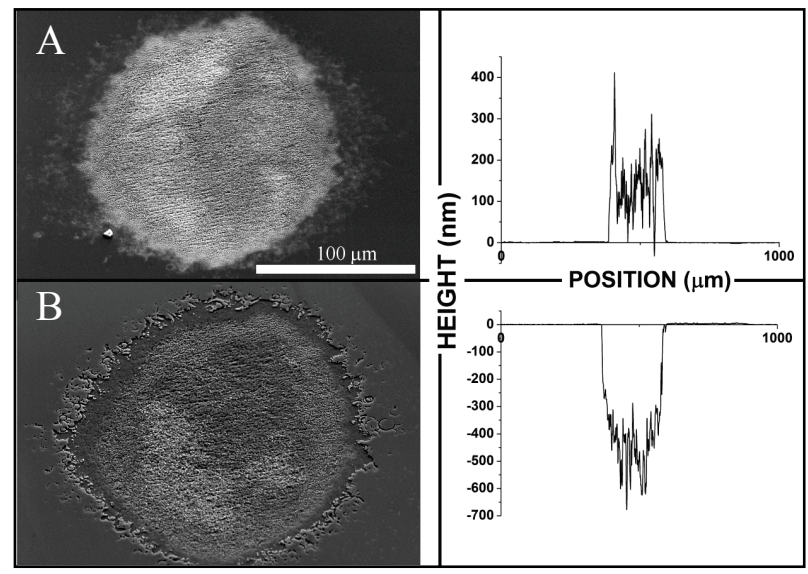

Fig. 6 SEM images (left column, sample tilt $45^{\circ}$ ), and corresponding profiles (right column) taken from areas, processed with $0.59 \mathrm{~J} / \mathrm{cm}^{2} \mathrm{~N}=100$ laser pulses in $\mathrm{KOH}$ solution. The samples were left in $\mathrm{KOH}$ solution after laser processing A: for 3 minutes and $\mathrm{B}$ : for 20 minutes

The SEM images and the corresponding depth profiles clearly show that laser processing itself does not result in etching, if the sample is immersed in the $\mathrm{KOH}$ solution during the processing time only. Holes as a result of laser ablation or etching were not detected by surface profile measurements, independently on the applied laser fluence, as shown in Fig. 7.

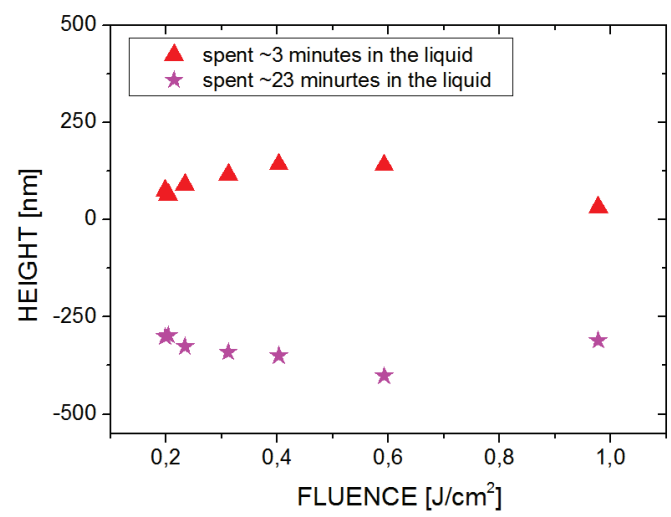

Fig. 7 Depths of ablated holes as a function of laser fluence, determined from profilometry measurements. Samples were exposed to etching in $\mathrm{KOH}$ solution at room temperature for 3 (triangles) and 23 minutes (stars). 
The evolution of the surface morphology with increasing pulse number is shown in Fig. 8. As can be seen after three pulses of laser, the treated surface became a bit rough. The value of roughness increased slightly when increasing the number of laser shots. During laser processing rough structures formed at or even slightly above the original surface level.
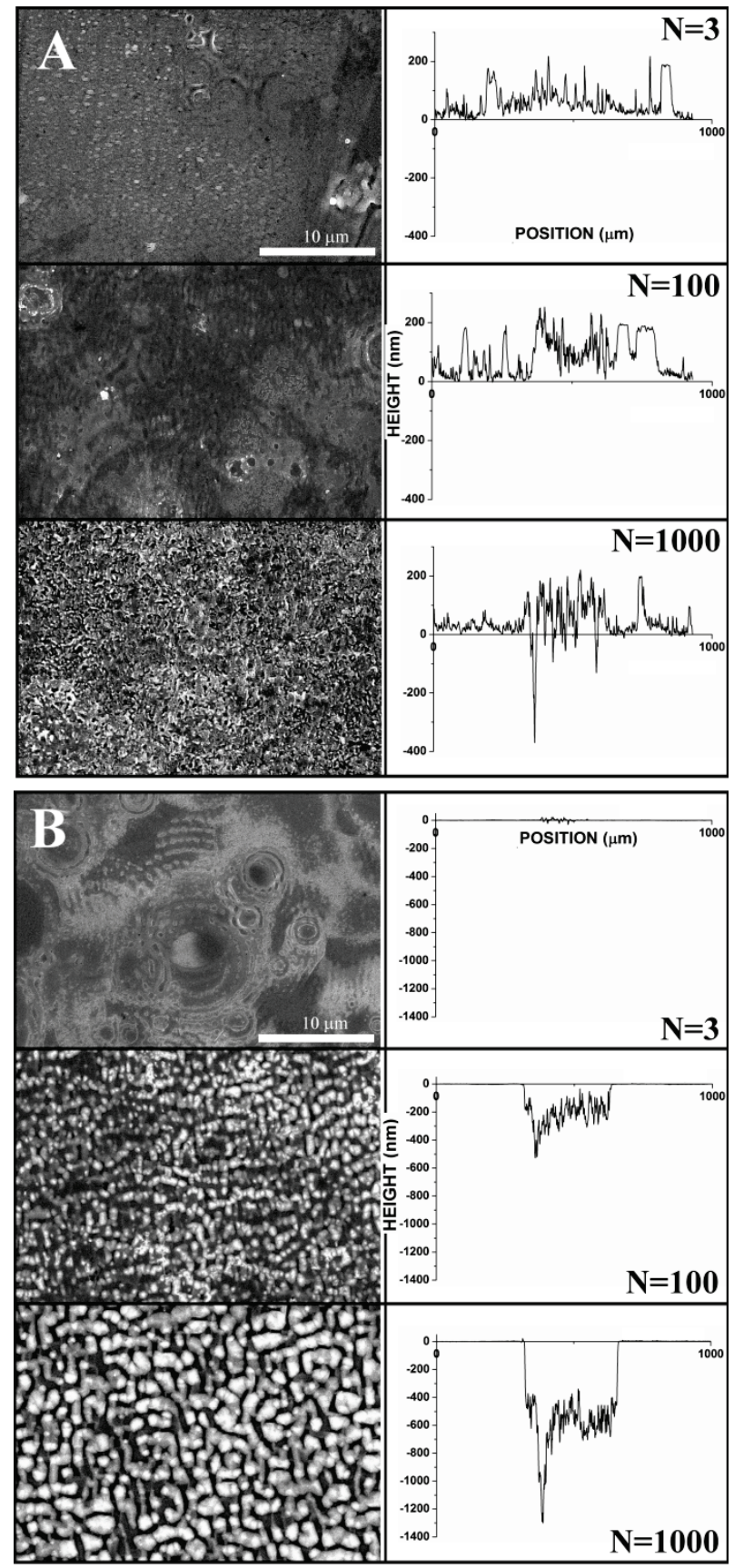

Fig. 8 SEM images (left column), and corresponding profiles (right column) taken from areas, processed with different $(3,100$ and 1000) number of pulses in $\mathrm{KOH}$ solution with $0.31 \mathrm{~J} / \mathrm{cm}^{2}$ fluence pulses. Samples were exposed to $\mathrm{KOH}$ for A) 3 and B) 18 minutes.

It is a surprising result that laser ablation or etching is not significant in $\mathrm{KOH}$ solution, because $\mathrm{KOH}$ is an etchant of $\mathrm{Si}$, which is widely applied in industry for etching or texturing silicon wafers. When keeping the laser processed samples in $\mathrm{KOH}$ solution for a longer time, $\mathrm{KOH}$ etching proceeded at those areas, where laser processing removed the native oxide layer of silicon. In Fig. 9 the depths of these holes, measured by profilometry, are also shown. It can be seen, that hole depth increases with increasing pulse number almost linearly. However, comparing the depths with that of the samples prepared in air or water, it can be concluded, that the depths of holes are much smaller. Thus etching is nearly independently proceeds at those areas, where native silicon dioxide does not protect the surfaces from $\mathrm{KOH}$ etching.

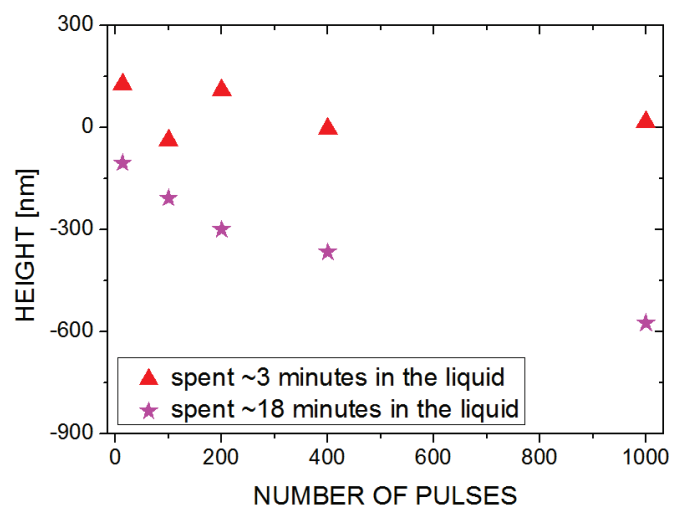

Fig. 9 Depths of ablated holes as a function of pulse number, determined from profilometry measurements. Samples were exposed to $\mathrm{KOH}$ solution after laser processing for 3 (triangles) and 18 (stars) minutes, respectively. The fluence of the pulses was $0.31 \mathrm{~J} / \mathrm{cm}^{2}$.

\section{Discussion}

When discussing multipulse ablation processes one has to consider, that already the second laser pulse ablates the surface which has been already modified by the first one. The forthcoming laser pulses are interacting with a continuously changing surface. SEM and profilometry investigations show that on the ablated areas surface roughness increases when increasing the laser fluence or the number of laser pulses. Increased surface roughness enhances light scattering. The interaction of incident beam with scattered and reflected light from surface structures during fs irradiation can result in interference $[17,18,20]$. In our experiments periodic structures were observed in the case of samples irradiated in air, which can be the consequence of this interference phenomenon [24].

Since the reflection of surfaces increases with increasing angle of incidence, the penetrating and therefore absorbed laser energy is the highest at surfaces which are perpendicular to the laser beam. After the first laser pulses only the bottom of the forming valleys will fulfill this condition, therefore the bottom of the valleys will absorb the highest intensity. This effect is further strengthened since multiple reflections appear at the side walls, which direct the light into the deeper parts of the structures. As a result the ablation becomes more intensive in the deepest parts, which result in deepening of the valleys, and increasing the roughness of the ablated surface. This process can only proceed in the mild ablation range [25], where the ablation 
rate is correlated to absorption penetration depth, and no thermal diffusion effects are effective in the excited volume.

If one considers the linear part of the absorption coefficient only, which can be measured at low level light intensity, the ablation of silicon by titanium sapphire laser cannot be an efficient process. The absorption penetration depth turns up in the range of $10 \mu \mathrm{m}$ at the wavelengths of titanium sapphire laser. If the energy of a single laser pulse, typically used in our experiments, is distributed in this thickness it may result in temperature increase, which causes the melting of silicon, but it is not efficient for vaporization and plasma formation. However, the formation of electron hole pairs during the onset of the laser pulse [3] drastically increases the absorption, as Drude absorption becomes effective. This and non-linear absorption result in the decrease of the absorbing zone, and as a consequence, high temperature electron gas is formed. This electron gas interacts with the silicon lattice and the ambient above it. The interaction firstly takes place with the outer valence electrons. Due to their collision, ionization takes place which causes plasma formation at the silicon-ambient interface, in accordance with our observations. The further relaxation processes involve further evaporation and chemical reactions at plasma-substrate interface, formation of mechanical waves (sound), and expansion of the gas. In the case of liquid ambient the gas phase forms a bubble, which later dissolves, collapses or can be removed by dynamic transport processes.

In the case of ablation in air, oxidation of high temperature silicon is very efficient in the plasma phase where oxygen ions and atoms are present. The plume expands and it is scattered on the particles of air. Therefore a part of the ablated material is deposited onto the silicon around the ablated area [23]. The surface is covered by a fine grain $\mathrm{SiO}_{2}$ powder as indicated by SEM and EDX. This type of deposit can prevent the surface from further ablation due to subsequent laser pulses. On one hand the thickness of the material increases, as shown by profilometry, and on the other hand, this transparent porous layer efficiently scatters the light and decrease the amount of absorbed laser intensity. This acts as a negative feedback, which results in narrowing of the ablated hole. The narrowing for ablation in air is striking when comparing depth profiles, obtained in liquid environments.

Such deposition around the ablated area cannot be observed in water, at least in not that large amount, like in air. This is probably due to the fluid flow caused by ablation processes. The observed light phenomenon in water was much stronger and whiter than in air. This suggests a higher temperature plasma formation, according to Boltzmann radiation law. The stronger radiation can be due to the higher temperatures and pressures, since the expansion of plasma is hindered in the incompressible fluid. As water has higher density than air, chemically active species (protons, hydrogen atoms, oxygen ions and atoms) can be formed in higher concentration. This can lead to greater chemical reactivity with $\mathrm{Si}$ ions, atoms and the hot surface. Laser treated surfaces in water were less oxidized than in the case of air. The majority of the resulting $\mathrm{SiO}_{2}$ is likely to remain in the form of a colloidal solution in water and moves away from treated zone by liquid flow. As a result no such negative feedback is present, like in the case of air.
The resulted etching is effective and the walls of the etched holes are abrupt. The depth profile is defined mainly by the plasma etching and in less extent by the intensity dependent ablation.

The most striking effect in our experimental results was that using $\mathrm{KOH}$ solution instead of water the etching was not accelerated, in fact it was inhibited. Etching in $\mathrm{KOH}$ is effective in aqueous solution. Light phenomenon in case of Si treatment in $\mathrm{KOH}$ solution was the strongest, indicating, that plasma effects took place there rather intensively. When considering plasma chemistry instead of chemistry in liquid case, it may resolve the ineffectiveness of etching in $\mathrm{KOH}$ solution during laser treatments. Potassium ions appear as a new constituent in high temperature plasma, which are rather reactive. As a consequence, new competing redox reactions may take place, as formation of potassium-oxide from atomic and ionic $\mathrm{K}$ and $\mathrm{O}$ radicals. Since electronegativity of $\mathrm{K}$ is lower than that of $\mathrm{Si}$, oxidation of $\mathrm{K}$ is more probable. This hypothesis is supported by the fact, that EDX indicated the lowest oxygen content (near to the detectable amount) in the treated areas. The etching of silicon in $\mathrm{KOH}$ solution proceeds after the laser treatment on those areas, where native oxide is removed. The observed etch rates $(\sim 0.016 \mu \mathrm{m} / \mathrm{min})$ is a good accordance with the etching rates determined by concentration and temperature conditions [26]. The slight increase in etching rate, when increasing the number of pulses, can be related to the formation of a defected rougher surface. A rougher surface has a higher area, where etching may proceed, thus resulting in higher etching rate.

\section{Conclusions}

We have shown that laser etching of Si surface with fs pulses can be performed more effectively in water than in air. The increase of the effectiveness is attributed to the incompressibility and to the larger density of water. The expansion of the plasma plume is slower than in air. Therefore the local temperature is increased and higher concentration of the reactants is ensured. Furthermore we have shown, that the application of a chemically active liquid, namely $\mathrm{KOH}$ solution does not necessarily help to increase the etching rate. Even it can stop the etching of Si surface, if it modifies plasma chemical reactions in a reverse way. However, it has been also shown that post-treatment in $\mathrm{KOH}$ solution allows the local etching of $\mathrm{Si}$ at predefined positions assigned to the laser irradiated areas.

\section{Acknowledgments}

The project was partially funded by TÁMOP-4.2.2.A11/1/KONV-2012-0047- "New functional materials and their biological and environmental answers" and is supported by the European Union and co-financed by the European Social Fund.

\section{References}

[1] D. Bäuerle: "Laser Processing and Chemistry" (Springer Verlag, Berlin, Heidelberg, 2011) p.279.

[2] Z. Toth, B. Hopp, J. Klebniczki, N. Kresz, Z. Bor, D. Anglos, C. Kalpouzos and C. Fotakis: Appl. Phys. A 79 (2004) 1287

[3] S. K. Sundaram and E. Mazur: Nature Materials 1 (2002) 217 
[4] A. Kruusing: Optics and Lasers in Engineering 41 (2004) 329

[5] R. Torres, V. Vervisch, M. Halbwax, T. Sarnet, P. Delaporte, M. Sentis, J. Ferreira, D. Barakel, S. Bastide, F. Torregrosa, H. Etienne and L. Roux: J.Opt.el. Adv. Mat. 12 (2010) 621

[6] O. Varlamova, F. Costache, J. Reif and M. Bestehorn: Appl. Surf. Sci. 252 (2006) 4702

[7] T.-H. Her, R. J. Finlay, C. Wu, S. Deliwala and E. Mazur:Appl. Phys. Lett., 73 (1998) 1673

[8] S. Besner, J.-Y. Degorce, A.V. Kabashin and M. Meunier: Appl. Surf. Sci. 247 (2005) 163

[9] S. Kontermann, T. Gimpel, A. L. Baumann, K.-M. Guenther and W. Schade: Energy Procedia 27 (2012) 390

[10] Y. Han and S.Qu: Chem. Phys. Lett. 495 (2010) 241

[11] W.C. Shen, C.W. Cheng, M.C. Yang, Y. Kozawa and S. Sato: J. Laser Micro/Nanoeng. 5 (2010) 229

[12] L. Ionel, C. P. Cristescu, F. Jipa, M. Enculescu, M. Radiou, R. Dabu, M. Zamfirescu and M. Ulmenau: OptoelectronicsandAdv.Mat. 4 (2010), 1920

[13] H. Liu, F. Chen, X. Wang, Q. Yang, H. Bian, J. Si and X. Hou: Thin Solid Films 518 (2010) 5188

[14] M. Ulmeanu, F. Jipa, C. Radu, M. Enculescu and M. Zamfirescu: Appl. Surf. Sci. 258, (2012) 9314.
[15] C. B. Schaffer, N. Nishimura, E. N. Glezer, A. M.-T. Kim and E. Mazur: Opt. Express, 10 (2002) 196-203

[16] M. Y. Shen, C. H. Crouch, J. E. Carey and E. Mazur: Appl. Phys. Lett., 85 (2004) 5694

[17] M. Shen, J. E. Carey, C. H. Crouch, M. Kandyla, H. A. Stone and E. Mazur: Nano Letters, 8 (2008) 2087

[18] K. Miyazaki, G. Miyaji: Physics Procedia 39 (2012) 674

[19] G. Daminelli, J. Krüger and W. Kautek: Thin Solid Films 467 (2004) 334

[20] G. Miyaji, K. Miyazaki, K. Zhang, T. Yoshifuji and J. Fujita: Optics Express 20 (2012) 14848

[21] G. Miyaji, K. Zhang, J. Fujita and K. Miyazaki: J. Laser Micro/Nanoeng. 7 (2012) 198

[22] B. Farkas and Z. Geretovszky: Appl. Surf. Sci. 252 (2006) 4728

[23] L. Égerházi, Z. Geretovszky, T. Csákó and T. Szörényi: Appl. Surf. Sci. 252 (2006) 4661

[24] M. Füle, J. Budai, Z. Szkiva, K. Sugioka, and L. Nánai: J. Laser Micro/Nanoeng. 9, 46-51 (2014)

[25] S. Nolte, C. Momma, H. Jacobs, A. Tünnermann, B. N. Chichkov, B. Wellegehausen and H. Welling: J. Opt. Soc. Am. B 14 (1997) 2716

[26] H. Seidel, L. Csepregi, A. Heuberger, and H. Baumgärtel: J. Electrochem. Soc. 137(1990) 3612

(Received: August 19, 2013, Accepted: April 10, 2014) 